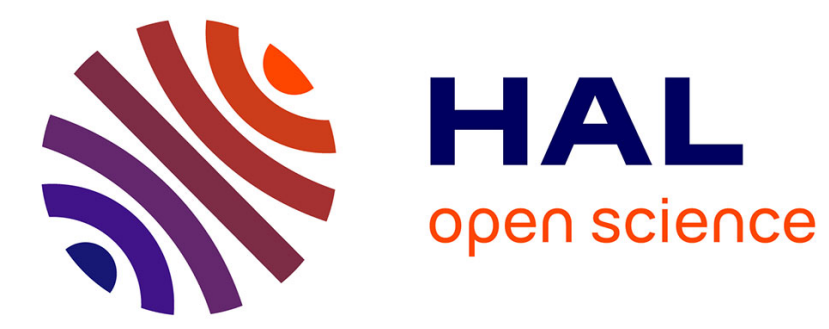

\title{
Do Astrocytes Play a Role in Intellectual Disabilities?
}

Noémie Cresto, Laure-Elise Pillet, Pierre Billuart, Nathalie Rouach

\section{To cite this version:}

Noémie Cresto, Laure-Elise Pillet, Pierre Billuart, Nathalie Rouach. Do Astrocytes Play a Role in Intellectual Disabilities?. Trends in Neurosciences, 2019, 42 (8), pp.518-527. 10.1016/j.tins.2019.05.011 . hal-02626510

\section{HAL Id: hal-02626510 \\ https://hal.science/hal-02626510}

Submitted on 25 Oct 2021

HAL is a multi-disciplinary open access archive for the deposit and dissemination of scientific research documents, whether they are published or not. The documents may come from teaching and research institutions in France or abroad, or from public or private research centers.
L'archive ouverte pluridisciplinaire HAL, est destinée au dépôt et à la diffusion de documents scientifiques de niveau recherche, publiés ou non, émanant des établissements d'enseignement et de recherche français ou étrangers, des laboratoires publics ou privés.

\section{다)(1) $(5$}

Distributed under a Creative Commons Attribution - NonCommercial| 4.0 International 


\section{Do astrocytes play a role in intellectual disabilities?}

$$
\text { Noémie Cresto }{ }^{1,2+} \text {, Laure Elise Pillet }{ }^{1,2,3+}, \text { Pierre Billuart }^{2 *} \text { and Nathalie Rouach }{ }^{1 *}
$$

${ }^{1}$ Neuroglial Interactions in Cerebral Physiopathology, Center for Interdisciplinary Research in Biology, Collège de France, CNRS UMR 7241, INSERM U1050, 75005 Paris, France

${ }^{2}$ Centre de Psychiatrie et de Neurosciences, INSERM U894, 75014 Paris, France

${ }^{3}$ Doctoral School N562, Paris Descartes University, Paris 75006, France

,$+ *$ Equally contributing authors

Correspondence to:

Rouach, N. (nathalie.rouach@college-de-france.fr)

Billuart, P. (pierre.billuart@inserm.fr)

\section{Keywords}

astrocytes, intellectual disability, Fragile X syndrom, Down syndrom, Rett syndrom, tripartite synapse

\section{Abstract}

Neurodevelopmental disorders, including those involving intellectual disability, are characterized by abnormalities in formation and functions of synaptic circuits. Whereas traditionally, research on synaptogenesis and synaptic transmission in health and disease focused on neurons, a growing number of studies have highlighted the role of astrocytes in this context. Tight structural and functional interactions of astrocytes and synapses indeed play important roles in brain functions, and the repertoire of astroglial regulations of synaptic circuits is large and complex. Recently, genetic studies of intellectual disabilities have underscored potential contributions of astrocytes in the pathophysiology of these disorders. Here we review how alterations of astrocytes' functions in disease may interfere with neuronal excitability and the balance of excitatory and inhibitory transmission during development, and contribute to intellectual disabilities. 


\section{Intellectual disabilities}

Intellectual Disability (ID, also called learning disability, mental retardation, or cognitive deficit) is defined by an overall Intelligence Quotient (IQ) lower than 70 associated with deficits in conceptual, social and practical adaptive skills with an onset before the age of 18 years. The clinical spectrum of cognitive deficit varies widely from non-syndromic ID to Autism Spectrum Disorder (ASD) and is estimated to affect 1 to $3 \%$ of the population. The causes of ID are heterogeneous and include genetic and/or environmental factors that influence the development and function of the central nervous system (CNS) during the pre-, peri-, or post-natal period. Genetic causes, including chromosomal abnormalities, such as in Down syndrome (DS), and monogenic causes, in Rett and Fragile X syndromes (RS and FXS, respectively), seem to be responsible for 40 to $50 \%$ of moderate to severe ID (IQ < 50). Environmental factors, including neurotoxic insults, maternal exposure to infections (toxoplasmosis, cytomegalovirus and rubella), or teratogenic molecules (alcohol), malnutrition and brain lesions during early development, primarily contribute to mild ID (50 $<I Q<70)$. In theory, genes that are involved in cases of ID in which CNS organization is preserved at a gross anatomical level (i.e., normal MRI scan), are likely to encode proteins that are necessary for neural circuit development, in a manner that ultimately contributes to impaired cognitive function. Though at first sight, it may appear that these proteins are very diverse, upon closer analysis, functional commonalities between these proteins emerge (e.g., transcription and chromatin remodeling factors, cell adhesion molecules, processing, modification, and transport of RNA, protein ubiquitination, and fatty acid metabolism function). Compelling evidence indicates that a major functional group of ID-related proteins corresponds to proteins that are enriched at synaptic compartments, and recent investigations point out the crucial role of these synaptic proteins in the regulation of dendritic spines morphogenesis and synaptic activity $[1,2]$.

Of note, new findings regarding the role of astrocytes in brain development and functions have emerged. These raise many questions regarding the contribution of neuroglial interactions in the pathogenesis of neurodevelopmental diseases. Here we review the alterations in the morphological and functional properties of astrocytes occurring in DS, RS and FXS and how this may affect neuronal development and functions. We also briefly 
address alterations and potential contributions of astrocytes in environmental forms of IDs (Box 1).

\section{Intrinsic astroglial alterations in ID?}

\section{Neuropathies: a restrictive vision of ID}

Expression of genes involved in ID, initially viewed as mainly confined to neurons, was recently described in astrocytes, suggesting that deficient astrocytes contribute to ID [3]. RNA sequencing technologies of brain cells $[4,5]$ revealed that most ID genes [6] are indeed expressed not only in neurons, but also in astrocytes (about $70 \%$ of ID genes). These genes encode for proteins enriched in mitochondria and lysosomes and that play roles in oxydoreduction functions known to be altered in several brain diseases[7,8]. Notably, FMRP and MeCP2, whose genes are mutated in FXS and RS respectively, as well as several genes of human chromosome 21 (HSA21) that are triplicated in DS (SOD1, DYRK1A, S100 $\beta$ and OLIG2), are expressed in astrocytes. Other glial cell types also express ID genes and show alterations in IDs, including FXS, RS and DS, and accordingly, these cell types may contribute to these disorders as well [9-13].

It is still unclear however whether ID proteins have similar or different functions when expressed in neurons or in astrocytes. ID proteins indeed may interact, regulate or target different sets of proteins or genes during development in a cell specific manner. In FXS, for instance, the FMRP protein regulates mGluR5 expression in astrocytes, but not in neurons. This leads to regulation of GLT1 glutamate transporter expression and associated glutamate uptake in astrocytes [14]. Notably, FMRP can also control glutamate transport in neurons, but via the direct regulation of the VGLUT1 vesicular transport 15].

Similarly, the MeCP2 protein, which binds to methylated DNA, increases or decreases expression of different sets of genes in neurons [16] and astrocytes [17,18] through chromatin structural changes [19]. Among the downregulated genes in astrocytes, two encode for membrane receptors that mediate calcium signaling (mGluR3R and S1P1R) and one for an ion channel involved in potassium uptake (Kir4.1) [20]. In addition, astrocytes from MeCP2 deficient mice show a reduction in the expression of stathmin-like 2, a protein involved in microtubule stability [21] and both mouse and human astrocytes showed altered microtubule dynamics and vesicular secretion [22]. Thus, the cell-specific functions of ID 
proteins are a great source of information that should lead to a better understanding of the contribution of astrocytes in ID.

\section{Abnormal astrocytic development and state}

Whereas most IDs and other related pathologies are presented as neurodevelopment disorders (with a focus on the neural compartment), the development of astrocytes is also affected, and changes in astrocyte number may impact the CNS development. Studies in DS showed an increase of astrocytes (more mature in their morphology) and radial glial cells in frontal lobe of fetuses at different stages [23]. Similarly, an increase of astrocytic differentiation from neuronal stem cells (NSC) has been observed in RS [24].

Recent evidence indicates that mutant astrocytes display an abnormal state in most IDs (Table 1 and Figure 1). Several astrocytic markers such as GFAP and S100 $\beta$ are indeed altered, and this is associated with a less complex morphology and thicker branches, suggesting astrocytic reactivity [25,26]. For instance, Rett patients have increased GFAP expression compared to matched controls [27], possibly because MeCP2 directly represses GFAP expression [28]. Increase in GFAP, but also $S 100 \beta$ expression, was also found in astrocytic cultures of MeCP2-deficient mice [29]. However, the modulation of GFAP expression is not as clear in the case of FXS, as both increase [30] or no change in GFAP expression [31,32] have been reported. In DS, dysregulated astrocytes were also observed in human brain tissue and differentiated cells from induced pluripotency stem cells (IPSCs) $[25,33]$. Interestingly, the human chromosome 21 carries the gene coding for $\$ 100 \beta$, which is triplicated in DS and leads to higher level of $S 100 \beta$ protein (75 times more) than control astrocytes [33]. Moreover, mutated astrocytes overexpress the GFAP protein (5 times more) although its gene is not located on the 21 chromosome, suggesting that astrocytes reactivity is not directly linked to the gene triplication. Whether astrocyte reactivity reported in some IDs applies to all astrocytes or subpopulations is currently unknown. It will thus be of interest to analyze the transcriptomic datasets of astrocytes in IDs and search, for instance, for markers of toxic A1 and trophic A2 astrocytes, as recently described [34].

Notably, dysregulation of astrocytic markers in IDs is associated with alterations in typical functional properties of astrocytes, such as glutamate sensing and clearance, as detailed below. 
A common feature of most IDs is altered neuronal morphology. Reduced dendritic branching, long thin immature spines and reduced spine motility have indeed been reported in FXS, RS and DS, suggesting that synaptic networks remain in an immature state [35-37]. Interestingly, recent data indicate that astrocytes are involved in the formation and maturation of neuronal networks by acting at multiple levels, such as dendritic growth, synaptogenesis, synapse maintenance and pruning [38,39]. These astroglial regulations of synaptic networks are likely altered in IDs, as suggested by several studies (Table 1). Deletion of FMRP in astrocytes in vitro inhibits neuronal growth as well as delays formation of excitatory synapses and dendritic arborization [40,41], while in vivo, it increases the density of immature dendritic spines [32]. Similarly in RS, astrocytes derived from iPSCs differentiation alter neuronal morphology, and in vivo, re-expression of MeCP2 in astrocytes from constitutive MeCP2-deficient mice rescues dendritic branching and number of excitatory synapses through a non-cell autonomous effect $[42,43]$. Finally, DS astrocytes cocultured with wild type neurons decrease spine density $[44,45]$. Yet, the mechanisms underlying astroglial dysregulation of synapses in IDs remain unclear. Several studies suggest that secreted factors are implicated in this context. Among these factors, thrombospondin-1 (TSP-1), known to stimulate excitatory synapse formation [46], is decreased in astrocytes from FX [47] and DS models [45]. In addition, conditioned media from wild type astrocytes or exogenous TSP1 restores in co-cultures spines and excitatory synaptic abnormalities of neurons $[45,47]$. Further work is needed to unravel the molecular determinants of astroglial regulations of synapses.

\section{Dysregulation of neuronal excitability due to astrocytic failure}

Astrocytes are involved in the clearance of extracellular glutamate through GLT1 and GLAST glutamate transporters. They also sense glutamate levels via metabotropic glutamate receptors (mGluRs), which mediate calcium release from intracellular stores. In FMRPdeficient mice, GLT1 and astrocytic mGluR5, as well as GLAST, are reduced during development, causing an excess of extracellular glutamate, which contributes to neuronal hyperexcitability [14,31]. By contrast, in cultured astrocytes from MeCP2 deficient mice, 
glutamate clearance is increased in response to extracellular glutamate rise, most likely due to higher expression of GLT1 and GLAST [29]. Somewhat similarly to MeCP2 deficient astrocytes, DS astrocytes express higher levels of GLAST, and also of mGluR5 than control astrocytes, however with no change in GLT1 expression, leading to a higher rate of glutamate uptake $[33,48]$. In vivo, contradictory observations have been reported regarding the levels of glutamate in DS [7]. Altogether, astroglial glutamate uptake is altered in most IDs (Table 1) but the alterations in extracellular glutamate do not seem to account for the cognitive deficits in ID.

Astrocytes also possess high permeability to potassium ions and are able to spatially redistribute the local excess of synaptic potassium through gap junctions in neighboring regions. Transient increases in extracellular potassium levels caused by neuronal activity are indeed buffered by astrocytes, which prevents network hyperactivity. Interestingly, several IDs including FXS and DS are associated with changes in neuronal excitability resulting from alteration in the expression or activity of several neuronal potassium channels [49-51]. Some of these channels, such as BK channels or KCNJ6 and KCNJ15, are expressed in astrocytes [52] and may therefore also be dysregulated in FXS and DS.

In addition, other non-neuronal potassium channels expressed in astrocytes and mediating most of the activity-dependent potassium uptake can also be dysregulated, as shown in RS (Table 1 and Figure 1). MeCP2 is indeed a positive regulator of the Kcnj10 gene which encodes the Kir4.1 channel [20], and whose expression is drastically reduced in MeCP2 deficient-astrocytes, resulting in disruption of extracellular potassium homeostasis [53]. This may contribute to the epileptic activity observed in about $70 \%$ of Rett patients [57]. Such astrocytic potassium channels may therefore represent a novel therapeutic target for ID.

Connexins, the astroglial gap junction proteins, are also known to contribute to glutamate and potassium extracellular homeostasis [54] and epileptic activity [55] and may thereby also be implicated in the alterations in neuronal excitability and seizures in IDs. Interestingly, mutations in Cx43 gene (GJA1), which codes the main gap junction channel subunit of astrocytes, can cause oculo-dento-digital dysplasia whose neurological manifestations include both ID and epilepsy [56]. 
Of note, beyond astroglial proteins whose expression is altered in ID and which contribute to epileptic activity such as Kir4.1 [57] and GLT1 [58] or Cx43 [55], astrocytes also express some ID genes which are functionally implicated in seizures. The interleukin-1 receptor accessory protein like 1 (IL1RAPL1) gene, for instance, which codes a synaptic adhesion protein, is an ID gene [59] which is also expressed in mouse astrocytes [5] and whose deletion has been recently associated with startle epilepsy in humans [60]. Furthermore, mutations in nuclear distribution protein nudE homolog 1 coded by the NDE1 ID risk gene [61], which is crucial for cell positioning during cortical development and is strongly expressed by astrocytes [5], increases susceptibility to convulsions in C. Elegans and can cause human cortical malformation termed lissencephaly associated with epilepsy [62].

\section{- Calcium signalling}

Astrocytes, unlike neurons, do not exhibit action potentials, but they do display intracellular calcium signaling, thought to represent their excitability. Such signaling occurs in response to several stimuli, and mediates various responses, including release of neuroactive molecules, which can regulate neuronal excitability or synaptic transmission. A couple of studies indicate that astroglial calcium signaling is enhanced in ID gene related pathologies (Table 1). For instance, in FX associated tremor/ataxia syndrome, astrocytes exhibit increased spontaneous asynchronous calcium oscillations [63]. In a DS mouse model, astrocytes basal calcium levels are also enhanced and evoked calcium responses are reduced likely due to an accumulation in organelles that may buffer cytosolic calcium [64]. Finally, aberrant calcium signaling also occurs in DS-patient-derived astroglia, with more frequent and increased spontaneous calcium fluctuations, independently of functional astroglial maturation [65]. The functional impact of the elevated astroglial calcium signaling still remains elusive, but the elevated calcium levels are likely to alter neuronal excitability, as reported for DS astroglia, where prominent calcium signaling reduces excitability of control co-cultured

\section{Excitation/Inhibition imbalance resulting from astroglial dysfunction?}

The balance between excitatory and inhibitory transmission (E/I) is commonly altered in FXS, RS and DS [37]. This effect may result from changes in the number of synapses or from 
regulation of ionic gradient and homeostasis altering the effect of neurotransmitters (Table 1 and Figure 1).

\section{- Reduction in excitatory synapses}

As mentioned above, astrocytes in IDs participate to the immature excitatory spines phenotype. This likely results in decreased excitatory transmission and altered $\mathrm{E} / \mathrm{I}$ balance. The overall astroglial control of $E / I$ balance is however still unclear, partly because few data are available regarding the role of astrocytes in the formation, maturation and elimination of inhibitory synapses $[66,67]$.

\section{- Regulation of the $\mathrm{Cl}^{-}$homeostasis}

Astrocytes have been shown to accelerate the developmental changes in chloride gradient of cultured neurons [68]. This suggests an additional mechanism that may control the E/I balance via regulation of the GABA switch occurring during early development, and which has been reported to be dysregulated in ID $[69,70]$.

While activation of $\mathrm{GABA}_{A}$ receptors in adult neurons induces an hyperpolarization due to an entry of chloride into neurons, their activation during early development is thought to lead to a depolarization resulting from a net release of chloride [71]. This GABA switch results from variations in the expression of NKCC1 (decrease) and KCC2 (increase), two chloride cotransporters. These co-transporters are present not only in neurons [72] but also in astrocytes, suggesting that glial cells may also participate to chloride homeostasis regulation [5]. Of note, both NKCC1 and KKC2 expression are dysregulated in IDs. Specifically, a delay in KCC2 expression occurs in humans and mouse model of RS as well as in FMRP deficient mice, suggesting a developmental delay in the switch to inhibition, which may also alter the excitation/inhibition balance during developmental critical periods $[69,70]$. Similarly, a dysregulation of the $\mathrm{Cl}^{-}$equilibrium leading to an excitatory effect of GABA was observed in the Ts65Dn mouse model of DS, in this case stemming from NKCC1 overexpression [73]. Interestingly, KCC2 and NKCC1 are strongly regulated by BDNF and its receptor $\operatorname{TrKB}[74,75]$ and BDNF levels are altered in FXS, RS and DS mouse models [76-78]. Since astrocytes are now recognized as an alternative source for BDNF expression and secretion [79], they may thereby also regulate the GABA switch.

\section{Concluding remarks and future perspectives}


Genetically-linked IDs, including DS, RS and FXS are now well recognized to present neuronal alterations at the synaptic level. More recently, glial dysfunctions have also been reported in such diseases, yet their contribution remains elusive. Only in a few cases, for instance FXS and RS, astrocyte dysfunctions have been directly shown to contribute to synaptic defects. These defects relate, in particular, to the formation of excitatory synapses, dendritic branching and the density of dendritic spines. However, the mechanisms underlying astroglial dysregulation of synapses in IDs remain to be further studied. In particular, do mutations in ID genes disrupt astroglial pathways involved in the physiological control of synapse formation, maintenance or pruning [80] ? Or do these mutations selectively activate other astroglial pathways which differentially regulate synapses?

In addition, whether astroglial dysfunctions also contribute to the cognitive decline and behavioral changes associated with synaptopathies is not yet clear . One study, in MeCP2deficient mice, reported that re-expression of MeCP2 in astrocytes significantly improved locomotion and anxiety levels, restored respiratory abnormalities to a normal pattern, and prolonged lifespan compared to globally null mice $[42,81]$. These data, to our knowledge, are the first to directly indicate that astrocytes, like neurons, may be integral components of the neuropathology of RS. This urges the study of astroglial contribution to behavioral changes in other synaptopathies, such as autism spectrum disorders. At least in one case, the principles as observed in MeCP2-deficient mice, do not seem to directly generalize: in FXS, re-expression of FRMP in astrocytes does not restore normal behavior, in particular related to motor learning [32]. But an involvement of a non-neuronal cell population does seem relevant, in this model, since deletion of FRMP in a large proportion of neurons $(\sim 60 \%)$ did not induce a behavioral phenotype [15]. Altogether, these data suggest that the behavioral alterations in FXS may result from the interactions between dysfunctional neurons and astrocytes, rather than from cell-specific defects. In fact, dysfunctions in IDs have typically been studied either in neurons or astrocytes, but little is currently known on the nature of the alterations in neuroglial interactions at the morphological and functional level. Studying the astroglial coverage of synapses using super resolution imaging, as well as bidirectional neuroglial calcium signaling at the tripartite synapse using cell-specific approaches is likely to provide further insights into the role of dysfunctional astrocyte-synapse interaction in IDs. 
One could envision that targeting pathways involved in neuroglial interactions, rather than solely neurons or astrocytes, may pave the way for novel alternative therapeutic approach in IDs. Since astrogliogenesis mainly occurs during the postnatal period of neurodevelopment, there might also be a timely temporal window for therapeutic intervention to improve these neuroglial interactions and compensate for some neuronal deficits acquired before birth.

Finally, at a more fundamental level, understanding how astrocyte dysfunctions alter the development of functional synaptic circuits, should provide new conceptual frameworks for mechanistic understanding of neurodevelopmental disorders such as ID, thus setting ground for future clinical investigations.

\section{Glossary}

\section{Intellectual disability}

Neurodevelopmental disorders characterized by a low intellectual quotient associated with deficits in adaptability including sociability, conceptualization, and practical skills prior to age of 18 .

\section{The tripartite synapse}

Revised concept of the synapse defined as a functional unit formed by the pre- and postsynaptic elements as well as the surrounding astrocyte. The astrocyte integrates the moment-to-moment synaptic activity via its ion channels, neurotransmitter receptors and transporters, and provides feedback modulation of neurotransmission through uptake or release of neuroactive substances.

\section{Gliotransmission}

Release of neuroactive substances such as glutamate, ATP, D-serine, or lactate from astrocytes to modulate neuronal activity.

\section{GABA switch}

Neurodevelopmental phenomenon where NKCC1 activity decreases whereas KCC2 activity increases, leading to an inversion of the $\mathrm{Cl}$ - gradient. This inversion explains the transition of the effects of GABA on neuronal activity from depolarizing during early development to hyperpolarizing at later stages.

\section{Channelopathy}


A term describing pathologies related to the dysfunction of one or more ion channels, leading to an alteration in ionic homeostasis and defects in neuronal transmission.

Box1. Astrocyte alterations in non-genetic forms of IDs.

Among IDs, the genetic forms have been the ones mostly studied thanks to the use of genetic animal models enabling to address cell-type specific roles. The involvement of astrocytes emerges however also in environmental forms of IDs, i.e. ones that can result for instance from malnutrition, infection and toxics exposure during early development. Severe prenatal malnutrition can result in a range of cerebral dysfunctions including ID. In a rat model of protein malnutrition, a delay in the emergence of GFAP expression and a reduction in astrocyte number has been observed, suggesting astrocytes as possible contributors to neurodevelopmental disorders [82]. Moreover, in a rat model of neonatal overnutrition, increases in primary projections of GFAP positive cells and in GFAP/vimentin levels suggest a modification in glial coverage. The levels of glucose (GLUT-1, GLUT-2) and glutamate transporters (GLAST and GLT-1), all expressed in astrocytes, are also increased in those rats, and associated with a decreased synaptic transmission, suggesting alterations in glucose and glutamate responses [83].

Another example where astrocytes seem to play a role is congenital cytomegalovirus (CMV) infection. Human CMV is the most common cause of congenital infections in developed countries. CMV infection causes serious neurodevelopmental sequelae, including ID. Lesions due to infection include ventricular dilation, white matter gliosis, atrophy and cortical malformations [84]. It has been shown that CMV can reduce the capacity of human neural cells to differentiate into astrocytes and thereby contribute to brain development abnormalities associated with congenital infection [85]. Astrocytes may also be involved in fetal alcohol spectrum disorder, a common cause of ID in the western world, causing cognitive and sensory impairments after birth [86]. The effects of fetal alcohol exposure (FAE) on the brain can vary throughout gestation according to the targeted neurodevelopmental stage and the corresponding dominant neuronal maturation event, progressing through proliferation, migration and differentiation. Alcohol exposure can strongly affect proliferation of glia and neuronal precursors, leading to microcephaly, hydrocephaly and ocular malformations [87]. FAE can also lead to abnormal migration of 
cortical neurons and later affects synaptogenesis, leading to persistent deficits in neuronal plasticity [88]. Of note, alcohol exposure at the neonatal stage can also induce alterations, such as reductions in cell number, maturation and survival of adult-born neurons and synaptic plasticity in the hippocampus [89]. The neuronal defects are accompanied by changes in glial development and function, including differentiation [90], impaired astrogliogenesis [91] and reduced glial cell survival [92]. Recently, it has also been demonstrated that FAE leads to a permanent change in the astrocyte secretome [86]. Astrocytes, by regulating circuit maturation, could thus participate in neuronal alterations underlying the spectrum of ID symptoms observed in FAE.

\section{Figure legend}

Figure 1. Astrocytes and the tripartite synapse in normal physiology versus ID. Schematic representation of normal physiological conditions (normal physiology, left panel) and putative pathophysiology in ID (ID, right panel) based on main alterations found in FXS, RS and DS. A number of common features have been reported to be altered in ID astrocytes, including changes in (1) astroglial complexity and reactivity; (2) synthesis and secretion of astroglial factors that support neuronal growth; (3) pruning of synapses by astrocytes leading to immature dendritic spine formation; (4) the synthesis and activity of astrocytic channels that regulates $\mathrm{K}^{+}$buffering, which prevents network hyperexcitability; and (5), expression of receptors (mGluR5) and transporters (GLT1, GLAST) enabling glutamate sensing and clearance. 


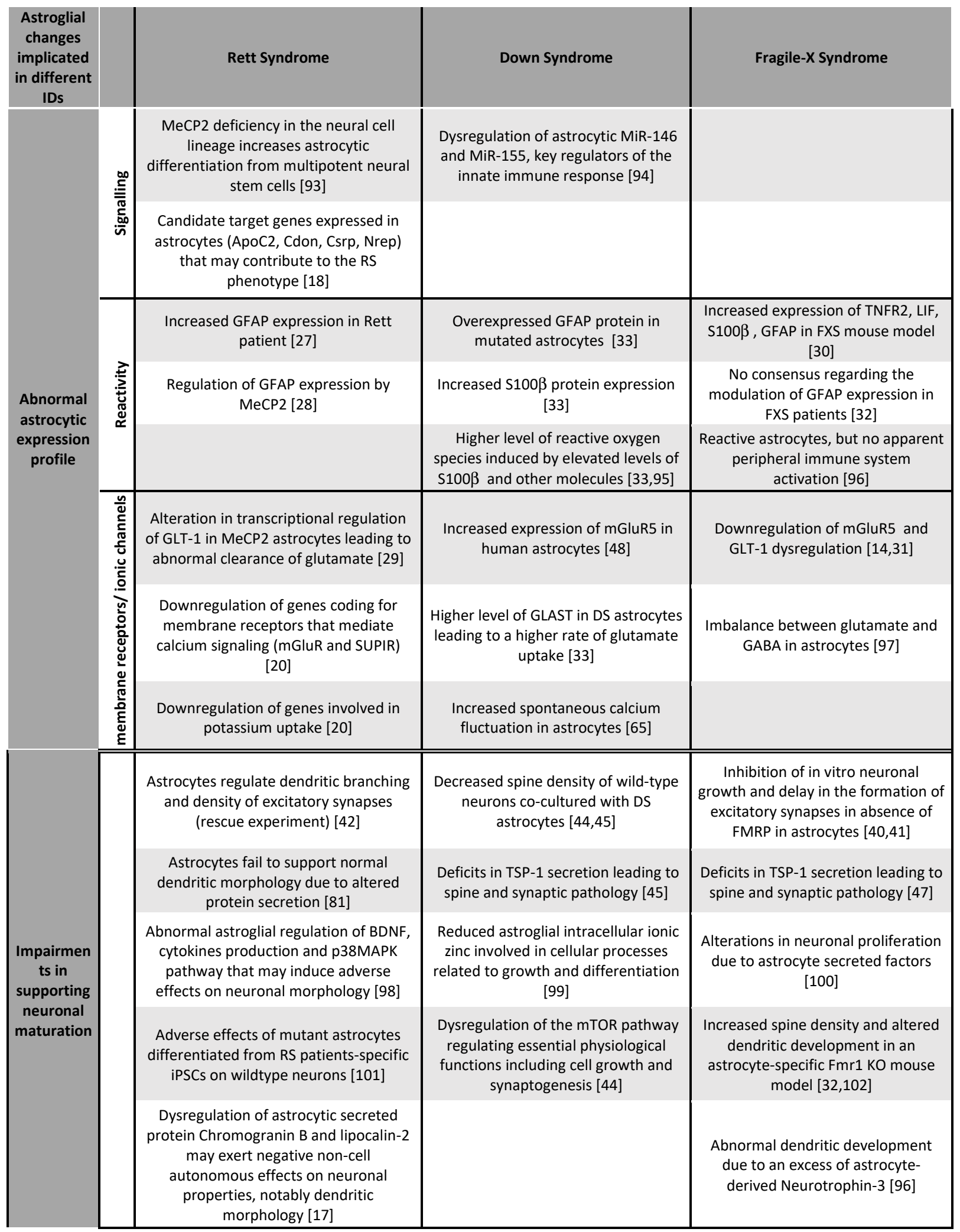

\section{Acknowledgements}

372 This work was supported by grants from the French National Agency for Research (grant \# 373 ANR-15-CE16-0019-01) to P.B. and N.R., from the Jerome-Lejeune Foundation (grant \# 1415) 
400

401

402

403

404

405

406

407

408

409

410

411

412

413

414

415

416

417

418

Union's Horizon 2020 research and innovation program (H2020-MSCA-ITN, grant \#722053, EU-GliaPhD) and Jerome-Lejeune Foundation (grant \#1535) to N.R. and from French

Research Ministry (BioSPC doctoral school, Paris Descartes University) to L.E. Pillet.

\section{References}

1 Dierssen, M. and Ramakers, G.J.A. (2006) Dendritic pathology in mental retardation: from molecular genetics to neurobiology. Genes Brain Behav. 2, 48-60

2 Gatto, C.L. and Broadie, K. (2010) Genetic controls balancing excitatory and inhibitory synaptogenesis in neurodevelopmental disorder models. Front. Synaptic Neurosci. 2, 4-4

3 McGann, J.C. et al. (2012) Astrocytes conspire with neurons during progression of neurological disease. Curr. Opin. Neurobiol. 22, 850-858

4 Zeisel, A. et al. (2015) Brain structure. Cell types in the mouse cortex and hippocampus revealed by single-cell RNA-seq. Science 347, 1138-1142

5 Zhang, Y. et al. (2014) An RNA-Sequencing Transcriptome and Splicing Database of Glia, Neurons, and Vascular Cells of the Cerebral Cortex. J. Neurosci. 34, 11929-11947

6 Lelieveld, S.H. et al. (2016) Meta-analysis of 2,104 trios provides support for 10 new genes for intellectual disability. Nat. Neurosci. 19, 1194-1196

7 Dossi, E. et al. (2018) Human astrocytes in the diseased brain. Brain Res. Bull. 136, 139156

8 Rama, K.R. and Kielian, T. (2016) Astrocytes and lysosomal storage diseases. Neuroscience 323, 195-206

9 Sharma, K. et al. (2018) MeCP2 in central nervous system glial cells: current updates. Acta Neurobiol. Exp. 78, 30-40

10 Olmos-Serrano, J.L. et al. (2016) Down Syndrome Developmental Brain Transcriptome Reveals Defective Oligodendrocyte Differentiation and Myelination. Neuron 89, 12081222

11 Pacey, L.K.K. et al. (2013) Delayed myelination in a mouse model of fragile X syndrome. Hum. Mol. Genet. 22, 3920-3930

12 Jawaid, S. et al. (2018) Alterations in CA1 hippocampal synapses in a mouse model of fragile X syndrome. Glia 66, 789-800

13 Xue, Q.-S. and Streit, W.J. (2011) Microglial pathology in Down syndrome. Acta Neuropathol. 122, 455

14 Higashimori, H. et al. (2013) Astroglial FMRP-dependent translational down-regulation of mGluR5 underlies glutamate transporter GLT1 dysregulation in the fragile X mouse. Hum. Mol. Genet. 22, 2041-2054

15 Amiri, A. et al. (2014) Analysis of Fmr1 Deletion in a Subpopulation of Post-Mitotic Neurons in Mouse Cortex and Hippocampus. Autism Res. 7, 60-71

16 Sugino, K. et al. (2014) Cell-type-specific repression by methyl-CpG-binding protein 2 is biased toward long genes. J. Neurosci. 34, 12877-12883

17 Delépine, C. et al. (2015) Astrocyte Transcriptome from the Mecp2(308)-Truncated Mouse Model of Rett Syndrome. Neuromolecular Med. 17, 353-363

18 Yasui, D.H. et al. (2013) MeCP2 modulates gene expression pathways in astrocytes. Mol. Autism 4, 3

19 Fasolino, M. and Zhou, Z. (2017) The Crucial Role of DNA Methylation and MeCP2 in Neuronal Function. Genes 8 
20 Pacheco, N.L. et al. (2017) RNA sequencing and proteomics approaches reveal novel deficits in the cortex of Mecp2-deficient mice, a model for Rett syndrome. Mol. Autism 8,56

21 Nectoux, J. et al. (2012) Altered microtubule dynamics in Mecp2-deficient astrocytes. J. Neurosci. Res. 90, 990-998

22 Delépine, C. et al. (2016) Altered microtubule dynamics and vesicular transport in mouse and human MeCP2-deficient astrocytes. Hum. Mol. Genet. 25, 146-157

23 Zdaniuk, G. et al. (2011) Astroglia disturbances during development of the central nervous system in fetuses with Down's syndrome. Folia Neuropathol. 49, 109-114

24 Andoh-Noda, T. et al. (2015) Differentiation of multipotent neural stem cells derived from Rett syndrome patients is biased toward the astrocytic lineage. Mol. Brain 8, 31

25 Jørgensen, O.S. et al. (1990) Neuronal plasticity and astrocytic reaction in Down syndrome and Alzheimer disease. J. Neurol. Sci. 98, 63-79

26 Nguyen, M.V.C. et al. (2012) MeCP2 is critical for maintaining mature neuronal networks and global brain anatomy during late stages of postnatal brain development and in the mature adult brain. J. Neurosci. 32, 10021-10034

27 Colantuoni, C. et al. (2001) Gene Expression Profiling in Postmortem Rett Syndrome Brain: Differential Gene Expression and Patient Classification. Neurobiol. Dis. 8, 847-865

28 Forbes-Lorman, R.M. et al. (2014) MeCP2 regulates GFAP expression within the developing brain. Brain Res. 1543, 151-158

29 Okabe, Y. et al. (2012) Alterations of gene expression and glutamate clearance in astrocytes derived from an MeCP2-null mouse model of Rett syndrome. PloS One 7, e35354

30 Pacey, L.K.K. et al. (2015) Persistent astrocyte activation in the fragile $\mathrm{X}$ mouse cerebellum. Brain Behav. 5, e00400

31 Higashimori, H. et al. (2016) Selective Deletion of Astroglial FMRP Dysregulates Glutamate Transporter GLT1 and Contributes to Fragile X Syndrome Phenotypes In Vivo. J. Neurosci. 36, 7079-7094

32 Hodges, J.L. et al. (2017) Astrocytic Contributions to Synaptic and Learning Abnormalities in a Mouse Model of Fragile X Syndrome. Biol. Psychiatry 82, 139-149

33 Chen, C. et al. (2014) Role of astroglia in Down's syndrome revealed by patient-derived human-induced pluripotent stem cells. Nat. Commun. 5, 4430

34 Zamanian, J.L. et al. (2012) Genomic analysis of reactive astrogliosis. J. Neurosci. Off. J. Soc. Neurosci. 32, 6391-6410

35 Grossman, A.W. et al. (2006) Hippocampal pyramidal cells in adult Fmr1 knockout mice exhibit an immature-appearing profile of dendritic spines. Brain Res. 1084, 158-164

36 Belichenko, N.P. et al. (2009) The "Down Syndrome Critical Region" Is Sufficient in the Mouse Model to Confer Behavioral, Neurophysiological, and Synaptic Phenotypes Characteristic of Down Syndrome. J. Neurosci. 29, 5938-5948

37 Benavides-Piccione, R. et al. (2004) On dendrites in Down syndrome and DS murine models: a spiny way to learn. Prog. Neurobiol. 74, 111-126

38 Allen, N.J. and Lyons, D.A. (2018) Glia as architects of central nervous system formation and function. Science 362, 181-185

39 Clarke, L.E. and Barres, B.A. (2013) Emerging roles of astrocytes in neural circuit development. Nat. Rev. Neurosci. 14, 311-321

40 Jacobs, S. and Doering, L.C. (2010) Astrocytes prevent abnormal neuronal development in the fragile $x$ mouse. J. Neurosci. 30, 4508-4514 
41 Jacobs, S. et al. (2010) Fragile X astrocytes induce developmental delays in dendrite maturation and synaptic protein expression. BMC Neurosci. 11, 132

42 Lioy, D.T. et al. (2011) A role for glia in the progression of Rett's syndrome. Nature 475, 497-500

43 Williams, E.C. et al. (2014) Mutant astrocytes differentiated from Rett syndrome patients-specific iPSCs have adverse effects on wild-type neurons. Hum. Mol. Genet. 23, 2968-2980

44 Araujo, B.H.S. et al. (2017) Down Syndrome iPSC-Derived Astrocytes Impair Neuronal Synaptogenesis and the mTOR Pathway In Vitro. Mol. Neurobiol. 55, 5962-5975

45 Garcia, O. et al. (2010) A role for thrombospondin-1 deficits in astrocyte-mediated spine and synaptic pathology in Down's syndrome. PloS One 5, e14200

46 Christopherson, K.S. et al. (2005) Thrombospondins are astrocyte-secreted proteins that promote CNS synaptogenesis. Cell 120, 421-433

47 Cheng, C. et al. (2016) Astrocyte-secreted thrombospondin-1 modulates synapse and spine defects in the fragile $X$ mouse model. Mol. Brain 9, 74

48 Iyer, A.M. et al. (2014) Metabotropic glutamate receptor 5 in Down's syndrome hippocampus during development: increased expression in astrocytes. Curr. Alzheimer Res. 11, 694-705

49 Brown, M.R. et al. (2010) Fragile X mental retardation protein controls gating of the sodium-activated potassium channel Slack. Nat. Neurosci. 13, 819-821

50 Deng, P.-Y. et al. (2013) FMRP Regulates Neurotransmitter Release and Synaptic Information Transmission by Modulating Action Potential Duration via BK Channels. Neuron 77, 696-711

51 Strumbos, J.G. et al. (2010) Fragile X mental retardation protein is required for rapid experience-dependent regulation of the potassium channel Kv3.1b. J. Neurosci. 30, 10263-10271

52 Seifert, G. et al. (2018) Diversity of astrocyte potassium channels: An update. Brain Res. Bull. 136, 26-36

53 Kahanovitch, U. et al. (2018) MeCP2 Deficiency Leads to Loss of Glial Kir4.1. eNeuro 5,

54 Pannasch, U. et al. (2011) Astroglial networks scale synaptic activity and plasticity. Proc. Natl. Acad. Sci. 108, 8467-8472

55 Bedner, P. et al. (2015) Astrocyte uncoupling as a cause of human temporal lobe epilepsy. Brain J. Neurol. 138, 1208-1222

56 De Bock, M. et al. (2013) Neurological manifestations of oculodentodigital dysplasia: a Cx43 channelopathy of the central nervous system? Front. Pharmacol. 4, 120.

57 Djukic, B. et al. (2007) Conditional Knock-Out of Kir4.1 Leads to Glial Membrane Depolarization, Inhibition of Potassium and Glutamate Uptake, and Enhanced ShortTerm Synaptic Potentiation. J. Neurosci. 27, 11354-11365

58 Tanaka, K. et al. (1997) Epilepsy and exacerbation of brain injury in mice lacking the glutamate transporter GLT-1. Science 276, 1699-1702

59 Carrié, A. et al. (1999) A new member of the IL-1 receptor family highly expressed in hippocampus and involved in X-linked mental retardation. Nat. Genet. 23, 25-31

60 Dinopoulos, A. et al. (2014) A case of startle epilepsy associated with IL1RAPL1 gene deletion. Pediatr. Neurol. 51, 271-274

61 Johnstone, M. et al. (2015) Copy Number Variations in DISC1 and DISC1-Interacting Partners in Major Mental Illness. Mol. Neuropsychiatry 1, 175-190 
62 Locke, C.J. et al. (2006) Genetic interactions among cortical malformation genes that influence susceptibility to convulsions in C. elegans. Brain Res. 1120, 23-34

63 Cao, Z. et al. (2013) Enhanced asynchronous $\mathrm{Ca}(2+)$ oscillations associated with impaired glutamate transport in cortical astrocytes expressing Fmr1 gene premutation expansion. J. Biol. Chem. 288, 13831-13841

64 Müller, W. et al. (1997) Impaired Ca-signaling in astrocytes from the Ts16 mouse model of Down syndrome. Neurosci. Lett. 223, 81-84

65 Mizuno, G.O. et al. (2018) Aberrant Calcium Signaling in Astrocytes Inhibits Neuronal Excitability in a Human Down Syndrome Stem Cell Model. Cell Rep. 24, 355-365

66 Chung, W.-S. et al. (2013) Astrocytes mediate synapse elimination through MEGF10 and MERTK pathways. Nature. 504, 394-400

67 Um, J.W. (2017) Roles of Glial Cells in Sculpting Inhibitory Synapses and Neural Circuits. Front. Mol. Neurosci. 10, 381

$68 \mathrm{Li}$, Y.-X. et al. (1998) Astrocytes regulate developmental changes in the chloride ion gradient of embryonic rat ventral spinal cord neurons in culture. J. Physiol. 509, 847-858

69 Duarte, S.T. et al. (2013) Abnormal expression of cerebrospinal fluid cation chloride cotransporters in patients with Rett syndrome. PloS One 8, e68851

70 Feldman, D. et al. (2016) Developmental Dynamics of Rett Syndrome. Neural Plast. 2016, 6154080

71 Ben-Ari, Y. et al. (1989) Giant synaptic potentials in immature rat CA3 hippocampal neurones. J. Physiol. 416, 303-325

72 Kahle, K.T. et al. (2008) Roles of the cation-chloride cotransporters in neurological disease. Nat. Rev. Neurol. 4, 490-503

73 Deidda, G. et al. (2014) Modulation of GABAergic transmission in development and neurodevelopmental disorders: investigating physiology and pathology to gain therapeutic perspectives. Front. Cell. Neurosci. 8, 119

74 Eftekhari, S. et al. (2014) BDNF modifies hippocampal KCC2 and NKCC1 expression in a temporal lobe epilepsy model. Acta Neurobiol. Exp. 74, 276-287

75 Rivera, C. et al. (2002) BDNF-induced TrkB activation down-regulates the $\mathrm{K}+-\mathrm{Cl}-$ cotransporter KCC2 and impairs neuronal Cl- extrusion. J. Cell Biol. 159, 747-752

76 Chang, Q. et al. (2006) The disease progression of Mecp2 mutant mice is affected by the level of BDNF expression. Neuron 49, 341-348

77 Incerti, M. et al. (2009) Role of brain-derived neurotrophic factor in Down syndrome. Am. J. Obstet. Gynecol. 201, S255

78 Louhivuori, V. et al. (2011) BDNF and TrkB in neuronal differentiation of Fmr1-knockout mouse. Neurobiol. Dis. 41, 469-480

79 Miklic, S. et al. (2004) Differences in the regulation of BDNF and NGF synthesis in cultured neonatal rat astrocytes. Int. J. Dev. Neurosci. 22, 119-130

80 Chung, W.-S. et al. (2015) Astrocytes Control Synapse Formation, Function, and Elimination. Cold Spring Harb. Perspect. Biol. 7, (9)

81 Ballas, N. et al. (2009) Non-cell autonomous influence of MeCP2-deficient glia on neuronal dendritic morphology. Nat. Neurosci. 12, 311-317

82 Naik, A.A. et al. (2017) Intra-generational protein malnutrition impairs temporal astrogenesis in rat brain. Biol. Open 6, 931-942

83 Fuente-Martín, E. et al. (2012) Leptin regulates glutamate and glucose transporters in hypothalamic astrocytes. J. Clin. Invest. 122, 3900-3913 
84 Cheeran, M.C.-J. et al. (2009) Neuropathogenesis of congenital cytomegalovirus infection: disease mechanisms and prospects for intervention. Clin. Microbiol. Rev. 22, 99-126

85 Odeberg, J. et al. (2007) Late human cytomegalovirus (HCMV) proteins inhibit differentiation of human neural precursor cells into astrocytes. J. Neurosci. Res. 85, 583593

86 Trindade, P. et al. (2016) Developmental alcohol exposure leads to a persistent change on astrocyte secretome. J. Neurochem. 137, 730-743

87 Miller, M.W. (1996) Effect of early exposure to ethanol on the protein and DNA contents of specific brain regions in the rat. Brain Res. 734, 286-294

88 Medina, A.E. (2011) Fetal alcohol spectrum disorders and abnormal neuronal plasticity. Neurosci. Rev. J. Bringing Neurobiol. 17, 274-287

89 Klintsova, A.Y. et al. (2012) Long-term consequences of developmental alcohol exposure on brain structure and function: therapeutic benefits of physical activity. Brain Sci. 3, 138

90 Rubert, G. et al. (2006) Ethanol exposure during embryogenesis decreases the radial glial progenitorpool and affects the generation of neurons and astrocytes. J. Neurosci. Res. 84, 483-496

91 Vallés, S. et al. (1996) Glial fibrillary acidic protein expression in rat brain and in radial glia culture is delayed by prenatal ethanol exposure. J. Neurochem. 67, 2425-2433

92 Holownia, A. et al. (1997) Ethanol-induced cell death in cultured rat astroglia. Neurotoxicol. Teratol. 19, 141-146

93 Andoh-Noda, T. et al. (2015) Differentiation of multipotent neural stem cells derived from Rett syndrome patients is biased toward the astrocytic lineage. Mol. Brain 8, 31

94 Arena, A. et al. (2017) Developmental Expression and Dysregulation of miR-146a and miR-155 in Down's Syndrome and Mouse Models of Down's Syndrome and Alzheimer's Disease. Curr. Alzheimer Res. 14, 1305-1317

95 Esposito, G. et al. (2008) Genomic and functional profiling of human Down syndrome neural progenitors implicates S100B and aquaporin 4 in cell injury. Hum. Mol. Genet. 17, 440-457

96 Yuskaitis, C.J. et al. (2010) Evidence of reactive astrocytes but not peripheral immune system activation in a mouse model of Fragile X syndrome. Biochim. Biophys. Acta 1802, 1006-1012

97 Wang, L. et al. (2016) Imbalance between Glutamate and GABA in Fmr1 Knockout Astrocytes Influences Neuronal Development. Genes 7, (8)

98 Maezawa, l. et al. (2009) Rett syndrome astrocytes are abnormal and spread MeCP2 deficiency through gap junctions. J. Neurosci. 29, 5051-5061

99 Ballestín, R. et al. (2014) Astrocytes of the murine model for Down Syndrome Ts65Dn display reduced intracellular ionic zinc. Neurochem. Int. 75, 48-53

100 Sourial, M. and Doering, L.C. (2016) Astrocyte-Secreted Factors Selectively Alter Neural Stem and Progenitor Cell Proliferation in the Fragile X Mouse. Front. Cell. Neurosci. 10, 126

101 Williams, E.C. et al. (2014) Mutant astrocytes differentiated from Rett syndrome patients-specific iPSCs have adverse effects on wild-type neurons. Hum. Mol. Genet. 23, 2968-2980

102 Jacobs, S. et al. (2016) Hippocampal neuronal subtypes develop abnormal dendritic arbors in the presence of Fragile X astrocytes. Neuroscience 324, 202-217 

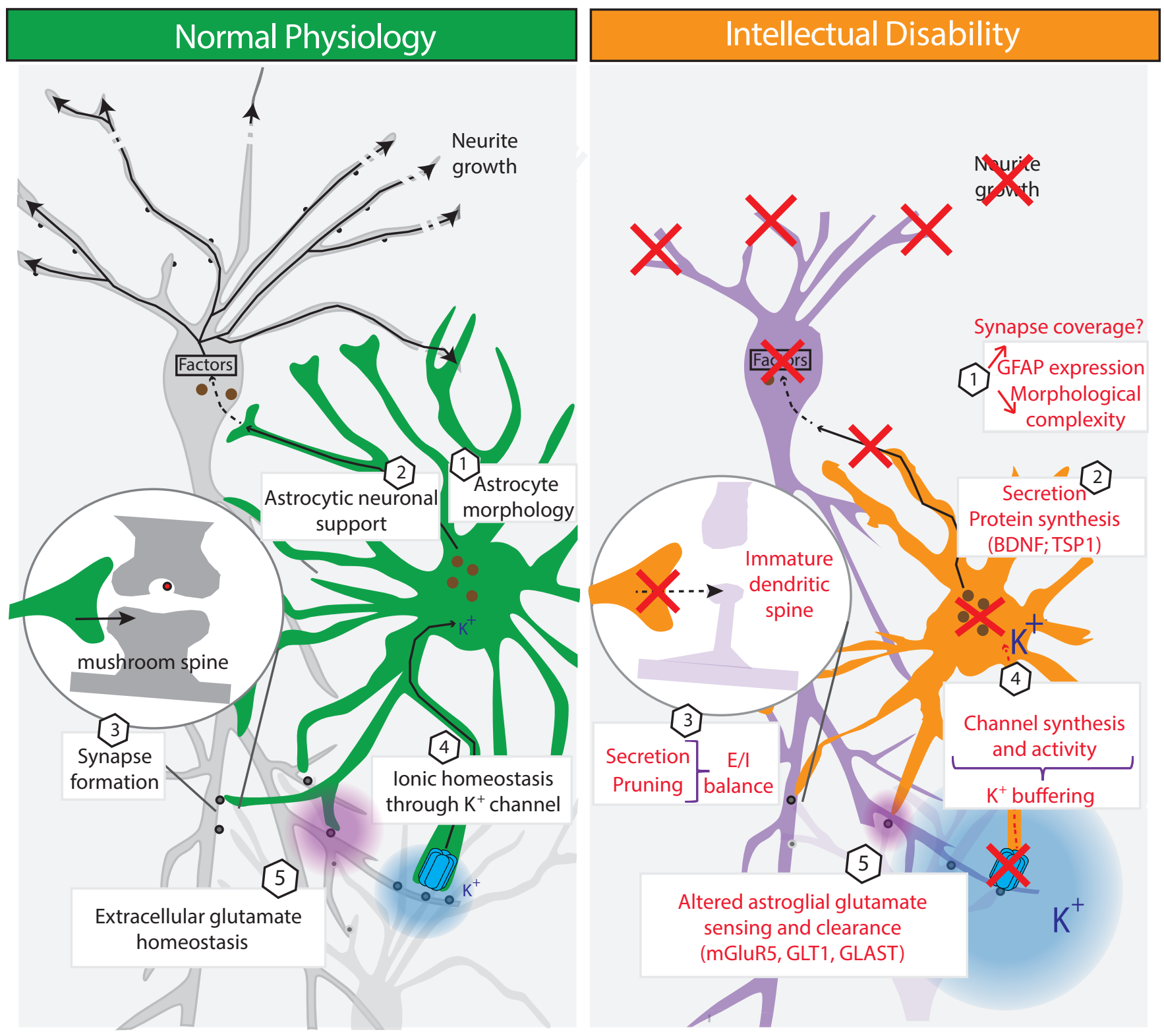